\title{
On Multiple Zagreb indices of Dendrimer Nanostars
}

\section{Mohammad Reza Farahani}

\author{
Department of Applied Mathematics of Iran University of Science and Technology (IUST), \\ Narmak, Tehran 16844, Iran
}

E-mail address: MrFarahani88@Gmail.com, Mr_Farahani@Mathdep.iust.ac.ir

Keywords: Molecular graphs; Dendrimer Nanostars; Zagreb indices; Multiple Zagreb indices

ABSTRACT. In this paper, we focus on the structure of an infinite class of Dendrimer Nanostars $D_{3}[n]$ ( $n \geq 0$ is infinite integer) and counting its First Multiple Zagreb index and Second Multiple Zagreb index. The Multiple Zagreb topological indices are equal to $P M_{l}(G)=\prod_{e=u v \in E(G)}\left(d_{v}+d_{v}\right)$ and $P M_{2}(G)=\prod_{e=u v \in E(G)}\left(d_{v} \times d_{v}\right)$, where $d_{v}$ is the degree of a vertex $v$.

\section{INTRODUCTION}

Let $G$ is an arbitrary simple, connected, graph, with the vertex set $V(G)$ and edge set $E(G)$. A graph is a collection of points and lines connecting a subset of them. The points and lines of a graph also called vertices and edges of the graph, respectively. A molecular graph is a simple graph such that its vertices correspond to the atoms and the edges to the bonds.

A connected graph is a graph such that there is a path between all pairs of vertices. If $e$ is an edge of $G$, connecting the vertices $u$ and $v$, then we write $e=u v$ and say " $u$ and $v$ are adjacent". The degree $d_{v}$ of a vertex $v \in V(G)$ is the number of vertices of $G$ adjacent to $v$. [1-4].

Mathematical chemistry is a branch of theoretical chemistry for discussion and prediction of the molecular graph using mathematical ways without referring to quantum mechanics [5-7]. Chemical graph theory is a branch of mathematical chemistry which applies graph theory to mathematical modeling of chemical phenomena. This theory plays a significant influence on the enlargement of the chemical sciences $[8,9]$.

A topological index is a real number associated with chemical constitution purporting for correlation of chemical structure with various physical properties, chemical reactivity or biological activity.

Wiener index [10-12] is the first topological index introduced by chemist H. Wiener in 1947. This index is defined as the sum of all topological distances between the pair of vertices. Here the topological distance between two vertices is the number of edges in the shortest path between them. This index is equal to

$$
W(G)=\frac{1}{2} \sum_{v \in V(G)} \sum_{u \in V(G)} d(v, u)
$$

where $d(u, v)$ is distance between the vertices $u$ and $v$ of the graph $G$.

One of the oldest topological index is the first Zagreb index an introduced by I. Gutman and N. Trinajstić on based degree of vertices of $G$ in 1972 [13]. The first and second Zagreb indices are defined as:

$$
\begin{gathered}
M_{l}(G)=\sum_{e=u v \in E(G)}\left(d_{v}+d_{v}\right) \text { or } M_{l}(G)=\sum_{v \in V(G)} d_{v}^{2} \\
M_{2}(G)=\sum_{e=u v \in E(G)}\left(d_{v} \times d_{v}\right)
\end{gathered}
$$

in which degree of a vertex $v$ of $G$ denoted by $d_{G}(v)$ (or $d_{v}$ for short). 
M. Ghorbani and his co-authors definition two new version of Zagreb indices of a graph $G$ in 2012 [14]. The Multiple Zagreb topological indices are defined as [15-18]:

$$
\begin{aligned}
& P M_{1}(G)=\prod_{e=u v \in E(G)}\left(d_{v}+d_{v}\right) \\
& P M_{2}(G)=\prod_{e=u v \in E(G)}\left(d_{v} \times d_{v}\right)
\end{aligned}
$$

In this paper, we focus on the structure of an infinite class of Dendrimer $D_{3}[n], \forall n \in \mathbb{N} \cup\{0\}(n \geq 1$ is infinite integer) and counting these new topological indices (the first and second Multiple Zagreb indices) for this molecular graph.

\section{RESULTS AND DISCUSSION}

Dendrimers are one of the main objects of Nano biotechnology. Here a dendrimer is a synthetic 3-dimensional macromolecule that is prepared in a step-wise fashion from simple branched monomer units, the nature and functionality of which can be easily controlled and varied. These molecular graphs are presented in many papers. We encourage the reader to consult papers [19-30] for further study on this topic. See Figure 1, which show the kind of $3^{\text {th }}$ growth of dendrimer.

In this paper, $D_{3}[n]$ denotes the $n^{\text {th }}$ growth of nanostar dendrimer for every infinite integer $n$ $(\forall n \in \mathbb{V} \cup\{0\})$ and we compute the First and Second Multiple Zagreb indices of this infinite class of Dendrimers.

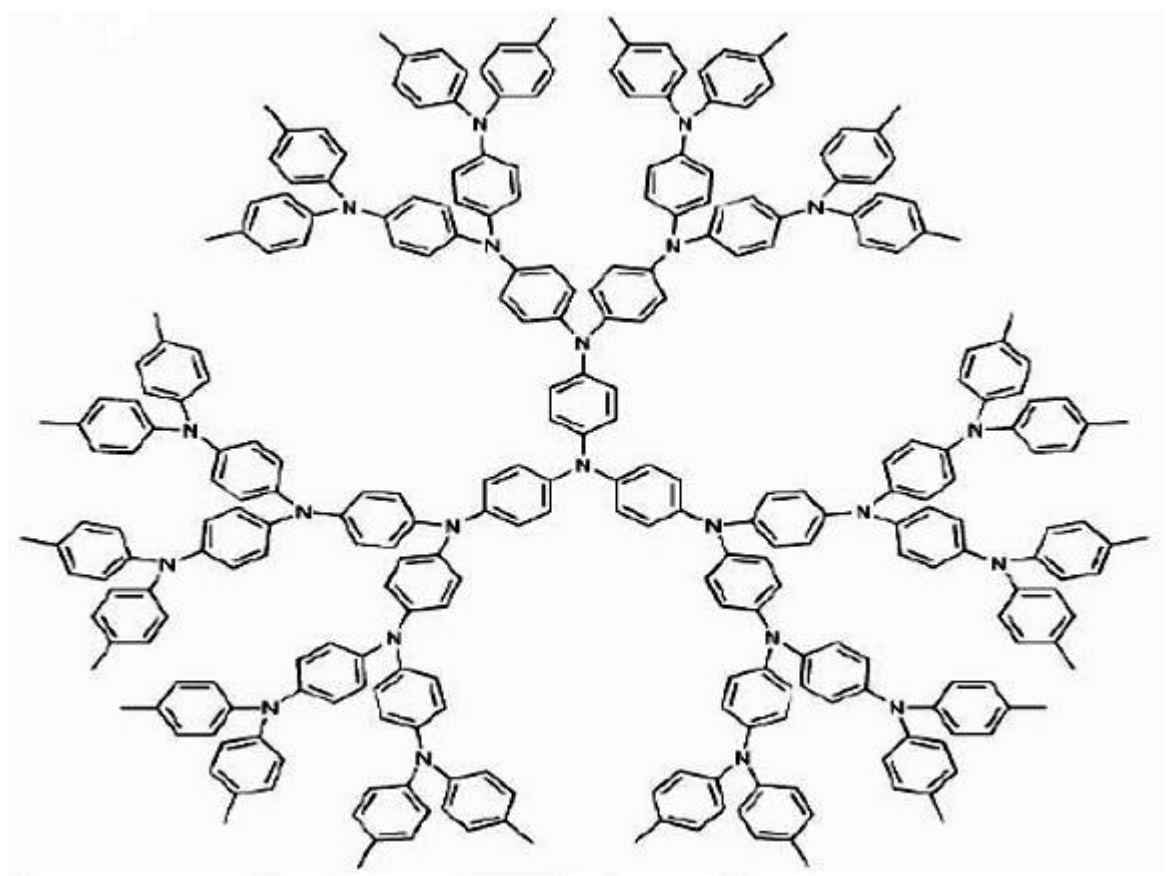

Figure 1. [27-30]: The 2-Dimensional of the $n^{\text {th }}$ growth of nanostar dendrimer $D_{3}[n], \forall n \in \mathbb{N} \cup\{0\}$.

Theorem 1. Let $D_{3}[n]$ be the Dendrimer Nanostars $\forall n \geq 0$. Then:

The First Multiple Zagreb index of $D_{3}[n]$ is equal to

$P M_{1}\left(D_{3}[n]\right)=(4)^{15\left(2^{n}\right)-6}+(5)^{12\left(2^{n+1}-1\right)}+(6)^{9\left(2^{n}\right)-6}$

The Second Multiple Zagreb index of $D_{3}[n]$ is equal to

$P M_{2}\left(D_{3}[n]\right)=(3)^{3\left(2^{n}\right)}+(2)^{12\left(2^{n+1}-1\right)}+(6)^{12\left(2^{n+1}-1\right)}+(3)^{18\left(2^{n}\right)-12}$

Proof of Theorem 1. Consider Dendrimer Nanostars $D_{3}[n](n \geq 0)$. In general case of this Dendrimer Nanostars, there are $\left|V\left(D_{3}[n]\right)\right|=7 \zeta_{n}+1=7 \times 3\left(2^{n+1}-1\right)=4\left(3\left(2^{n+1}\right)-5\right)$ vertices/atoms and 
$\left|E\left(D_{3}[n]\right)\right|=8 \zeta_{n}=24\left(2^{n+1}-1\right)$ edges/bonds. Such that $\zeta_{n}=3 \sum_{i=0}^{n}\left(2^{i}\right)=3\left(\frac{2^{n+1}-1}{2-1}\right)=3\left(2^{n+1}-1\right)$ is the number of leafs $\left(C_{6}\right)$ in the $n^{\text {th }}$ growth of Dendrimer Nanostar $D_{3}[n], \quad \forall n \geq 0$ (see Figure 1 and refernces [2730]).

Now, by using the results from reference [27-30] and the structure of Dendrimer Nanostar $D_{3}[n]$ in Figure 1, one can see that there are $3\left(2^{n}\right)$ vertices of $D_{3}[n]$ with degree $1,12\left(2^{n+1}-1\right)$ vertices of $D_{3}[n]$ with degree 2 and $15\left(2^{n}\right)$ vertices of $D_{3}[n]$ with degree three.

In other words, there are three vertex partitions of $V\left(D_{3}[n]\right)$ as follow

- $\quad V_{1}\left(D_{3}[n]\right)=\left\{v \in V\left(D_{3}[n]\right) \mid d_{v}=1\right\} \rightarrow\left|V_{1}\left(D_{3}[n]\right)\right|=v_{1}=3\left(2^{n}\right)$

- $\quad V_{2}\left(D_{3}[n]\right)=\left\{v \in V\left(D_{3}[n]\right) \mid d_{v}=2\right\} \rightarrow\left|V_{2}\left(D_{3}[n]\right)\right|=v_{2}=12\left(2^{n+1}-1\right)$

- $\quad V_{3}\left(D_{3}[n]\right)=\left\{v \in V\left(D_{3}[n]\right) \mid d_{v}=3\right\} \rightarrow\left|V_{3}\left(D_{3}[n]\right)\right|=v_{3}=15\left(2^{n}\right)$

This implies that four edge partitions of $E\left(D_{3}[n]\right)$ in following list [27-30]:

- $E_{2+2} \cup E_{1+3}=\left\{u v \in E\left(D_{3}[n]\right) \mid\left(d_{u}=d_{v}=2\right)\right.$ or $\left.\left(d_{u}=1 \& d_{v}=3\right)\right\} \rightarrow\left|E_{4}\right|=2 \zeta_{n}+3\left(2^{n}\right)=15\left(2^{n}\right)-6$

- $E_{2+3}=\left\{e=u v \in E\left(D_{3}[n]\right) \mid d_{u}=3 \& d_{v}=2\right\} \rightarrow\left|E_{5}\right|=4 \zeta_{n}=12\left(2^{n+1}-1\right)$

- $E_{3+3}=\left\{e=u v \in E\left(D_{3}[n]\right) \mid d_{u}=d_{v}=3\right\} \rightarrow\left|E_{6}\right|=2 \zeta_{n}-3\left(2^{n}\right)=9\left(2^{n}\right)-6$

- $E_{3}{ }^{*}=\left\{u v \in E\left(D_{3}[n]\right) \mid d_{u}=1 \& d_{v}=3\right\} \rightarrow\left|E_{3}{ }^{*}\right|=V_{1}\left(D_{3}[n]\right)=3\left(2^{n}\right)$

- $E_{4}{ }^{*}=\left\{u v \in E\left(D_{3}[n]\right) \mid d_{u}=d_{v}=2\right\} \rightarrow\left|E_{4}{ }^{*}\right|=2 \zeta_{n}=6\left(2^{n+1}-1\right)$

- $E_{6}{ }^{*}=\left\{u v \in E\left(D_{3}[n]\right) \mid d_{u}=3 \& d_{v}=2\right\} \rightarrow\left|E_{6}{ }^{*}\right|=\left|E_{5}\right|=4 \zeta_{n}=12\left(2^{n+1}-1\right)$

- $E_{9}{ }^{*}=\left\{u v \in E\left(D_{3}[n]\right) \mid d_{u}=d_{v}=3\right\} \rightarrow\left|E_{9}{ }^{*}\right|=\left|E_{6}\right|=2 \zeta_{n}-3\left(2^{n}\right)=9\left(2^{n}\right)-6$

Now, we have following computations for the first and second Multiple Zagreb indices of the $n^{\text {th }}$ growth of Dendrimer Nanostar $D_{3}[n], \forall n \geq 0$.

$$
\begin{aligned}
P M_{l}\left(D_{3}[n]\right) & =\prod_{u v \in E\left(D_{3}[n]\right)}\left(d_{v}+d_{u}\right) \\
& =\prod_{u v \in E_{4}}\left(d_{v}+d_{u}\right)+\prod_{u v \in E_{5}}\left(d_{v}+d_{u}\right)+\prod_{u v \in E_{6}}\left(d_{v}+d_{u}\right) \\
& =\sum_{i=4,5,6}(i)^{|E i|} \\
& =(4)^{\left|E_{4}\right|}+(5)^{\left|E_{5}\right|}+(6)^{\left|E_{6}\right|} \\
& =(4)^{15\left(2^{n}\right)-6}+(5)^{12\left(2^{n+1}-1\right)}+(6)^{9\left(2^{n}\right)-6}
\end{aligned}
$$

$$
\begin{aligned}
P M_{2}\left(D_{3}[n]\right) & =\prod_{u v \in E\left(D_{3}[n]\right)}\left(d_{v} \times d_{u}\right) \\
& =\prod_{u v \in E_{3}^{*}}\left(d_{v} \times d_{u}\right)+\prod_{u v \in E_{4}^{*}}\left(d_{v} \times d_{u}\right)+\prod_{u v \in E_{6}^{*}}\left(d_{v} \times d_{u}\right)+\prod_{u v \in E_{9}^{*}}\left(d_{v} \times d_{u}\right) \\
& =\sum_{j=3,4,6,9}(j)^{\left|E j^{*}\right|} \\
& =(3)^{E_{3}^{*} \mid}+(4)^{\left|E_{4}^{*}\right|}+(6)^{\left|E_{6}^{*}\right|}+(9)^{\left|E_{9}^{*}\right|} \\
& =(3)^{3\left(2^{n}\right)}+(2)^{12\left(2^{n+1}-1\right)}+(6)^{12\left(2^{n+1}-1\right)}+(3)^{18\left(2^{n}\right)-12}
\end{aligned}
$$

And these completed the proof of the Theorem 1 


\section{CONCLUSION}

In this paper, we counting two topological indices of an infinite class of Dendrimer Nanostars $D_{3}[n](\forall n \in \mathbb{N})$. The First Multiple Zagreb and Second Multiple Zagreb indices maybe useful to surveying the connected structure of Nanostars, Nanotubes and other Nanostructures, which have relation with degrees of its vertices latoms.

\section{References}

[1] D.B. West. An Introduction to Graph Theory. Prentice-Hall. (1996).

[2] R. Todeschini and V. Consonni. Handbook of Molecular Descriptors. Wiley, Weinheim. (2000).

[3] N. Trinajstić. Chemical Graph Theory. CRC Press, Bo ca Raton, FL. (1992).

[4] I. Gutman, N. Trinajstc. Chemical Physics Letters. 17, (1972). 535.

[5] M. V. Diudea, (Ed.), QSPR/QSAR Studies by Molecular Descriptors, NOVA, New York, 2001 .

[6] M. V. Diudea, I. Gutman and L. Jäntschi, Molecular Topology, NOVA, New York, 2002.

[7] M. V. Diudea, M. S. Florescu and P. V. Khadikar, Molecular Topology and Its Applications, EFICON, Bucharest, 2006.

[8] I. Gutman and O. E. Polansky, Mathematical Concepts in Organic Chemistry, SpringerVerlag, New York, 1986.

[9] M. A. Johnson and G. M. Maggiora, Concepts and Applications of Molecular Similarity, Wiley Interscience, New York, 1990.

[10] H. Wiener. Structural determination of paraffin boiling points. J. Am. Chem. Soc. 69, (1947), 17.

[11] A. Dobrynin, R. Entringer and I. Gutman. Acta Appl. Math. 66, (2001), 211.

[12] A. Dobrynin, I. Gutman, S. Klavzar and P. Zigert. Acta Appl. Math. 72, (2002), 247.

[13] I. Gutman and N. Trinajstić, Graph theory and molecular orbitals, Total $\pi$ - electron energy of alternant hydrocarbons, Chem. Phys. Lett., 1972, 17, 535-538.

[14] M. Ghorbani and N. Azimi. Note on multiple Zagreb indices. Iranian Journal of Mathematical Chemistry, 3(2), (2012), 137-143.

[15] I. Gutman and M. Ghorbani, Some properties of the Narumi-Katayama index, Appl. Math. Lett., 2012, 25, 1435-1438.

[16] M. Ghorbani, M. Songhori and I. Gutman, Modified Narumi-Katayama index, Kragujevac J. Sci., 2012, 34, 57-64.

[17] T. Došlić, On Discriminativity of Zagreb Indices, Iranian J. Math. Chem., 2012, 3(1), 25-34.

[18] M. Eliasi, A. Iranmanesh, I. Gutman. Multiplicative Versions of First Zagreb Index. MATCH Commun. Math. Comput. Chem. 68 (2012) 217-230.

[19] G.R. Newkome, C.N. Moorefield and F. Vogtlen, Dendrimer and Dendrons. Concepts, Syntheses, Applications. (Wiley-VCH Verlag Gmbh \& Co. Kgaa), (2002).

[20] A. Heydari, B. Taeri. MATCH Commun Math Comput Chem, 57, 463 (2007).

[21] M. Eliasi, B. Taeri, MATCH Commun Math Comput Chem, 59, 437 (2008).

[22] A.R. Ashrafi and P. Nikzad. Connectivity index of the family of Dendrimer Nanostars. Digest. J. Nanomater. Bios. 4, (2), 269-273. (2009). 
[23] A.R. Ashrafi and P. Nikzad. Kekule index and bounds of energy for Nanostar dendrimers. Digest. J. Nanomater. Bios. 4, (2), 383-388. (2009).

[24] A. Karbasioun and Ali Reza Ashrafi. Wiener and detour indices of a new type of nanostar dendrimers. Macedonian journal of chemistry and chemical engineering. 28(1), 49-54 (2009).

[25] S. Alikhani and M.A. Iranmanesh. Eccentric connectivity polynomials of an infinite family of dendrimer. Digest. J. Nanomater. Bios. 6(1), 256-257. (2011).

[26] M. Golriz, M.R. Darafsheh and M.H. Khalifeh. The Wiener, Szeged and PI-indices of a phenylazomethine dendrimer. Digest. J. Nanomater. Bios. 6(4), 1545-1549. (2011).

[27] M.R. Farahani. Fourth atom-bond connectivity index of an infinite class of Nanostar Dendrimer $D_{3}[n]$. Journal of Advances in Chemistry. 4(1), (2013), 301-305.

[28] M.R. Farahani. Computing Fifth Geometric-Arithmetic Index of Dendrimer Nanostars. Advances in Materials and Corrosion. 1, (2013), 62-64

[29] M.R. Farahani, The Zagreb Indices and Zagreb Polynomials of an infinite class of Dendrimer Nanostars. Submitted for publish. (2015).

[30] M.R. Farahani, Some Connectivity index of an infinite class of Dendrimer Nanostars. Journal of Applied Physical Science International (IK press), 2015, In press. 